\title{
Resonant hopping of a robot controlled by an artificial neural oscillator
}

\author{
Evan H Pelc ${ }^{1}$, Monica A Daley ${ }^{2,3}$ and Daniel P Ferris ${ }^{1,2,4}$ \\ ${ }^{1}$ Department of Biomedical Engineering, University of Michigan, Ann Arbor, MI 48109, USA \\ ${ }^{2}$ Division of Kinesiology, University of Michigan, Ann Arbor, MI 48109, USA \\ ${ }^{3}$ Structure and Motion Laboratory, Royal Veterinary College, Hatfield, Hertfordshire, AL9 7TA, UK \\ ${ }^{4}$ Department of Physical Medicine and Rehabilitation, University of Michigan, Ann Arbor, MI 48109, \\ USA \\ E-mail: ferrisdp@umich.edu
}

Received 14 July 2007

Accepted for publication 22 February 2008

Published 27 March 2008

Online at stacks.iop.org/BB/3/026001

\begin{abstract}
The bouncing gaits of terrestrial animals (hopping, running, trotting) can be modeled as a hybrid dynamic system, with spring-mass dynamics during stance and ballistic motion during the aerial phase. We used a simple hopping robot controlled by an artificial neural oscillator to test the ability of the neural oscillator to adaptively drive this hybrid dynamic system. The robot had a single joint, actuated by an artificial pneumatic muscle in series with a tendon spring. We examined how the oscillator-robot system responded to variation in two neural control parameters: descending neural drive and neuromuscular gain. We also tested the ability of the oscillator-robot system to adapt to variations in mechanical properties by changing the series and parallel spring stiffnesses. Across a 100-fold variation in both supraspinal gain and muscle gain, hopping frequency changed by less than $10 \%$. The neural oscillator consistently drove the system at the resonant half-period for the stance phase, and adapted to a new resonant half-period when the muscle series and parallel stiffnesses were altered. Passive cycling of elastic energy in the tendon accounted for 70-79\% of the mechanical work done during each hop cycle. Our results demonstrate that hopping dynamics were largely determined by the intrinsic properties of the mechanical system, not the specific choice of neural oscillator parameters. The findings provide the first evidence that an artificial neural oscillator will drive a hybrid dynamic system at partial resonance.
\end{abstract}

(Some figures in this article are in colour only in the electronic version)

\section{Introduction}

In hopping, running and trotting gaits, humans and all terrestrial animals use a similar 'bouncing' motion for economic locomotion [1-3]. A simple model describes the fundamental characteristics of these bouncing gaits remarkably well. The model is a hybrid dynamic system, comprising spring-mass dynamics during stance and ballistic motion during the aerial phases [4]. The stance phase requires the majority of muscle force and work to redirect the motion of the body. Consequently, most of the metabolic energy cost of locomotion is also associated with stance [5-8]. Spring-mass dynamics during stance can improve locomotor economy by cycling mechanical energy in elastic tissues and minimizing active muscle work [1,9]. In particular, long tendons of distal limb muscles, such as the Achilles tendon in humans, play a key role in storing and returning elastic energy during bouncing gaits [10].

Humans can adjust their overall effective leg stiffness to hop or run at different stride frequencies [11, 12], but they have a preferred leg stiffness when allowed to freely choose their movement dynamics [11, 13]. When human hopping or running is perturbed by compliant surfaces (i.e. series compliance) [14-16] or elastic leg braces (i.e. parallel compliance) [17], subjects adjust leg stiffness to maintain the same overall effective system stiffness. This results in a similar contact period across conditions. Some have argued that the contact period choice is related to neural reflex dynamics 
$[18,19]$, but other studies have pointed toward a mechanical explanation [20-22]. When a damped spring-mass system is rebounding off the ground, there is a time of contact with the surface that requires the least amount of energy input to maintain steady state because it takes advantage of the resonant frequency of vibration [22]. Driving an oscillating mechanical system at its natural frequency requires less mechanical energy input than driving the system at alternative frequencies. The relative importance of mechanical and neural factors in shaping hopping and running dynamics is unclear.

The neuromechanical control of locomotion is facilitated by rhythmic neural networks known as central pattern generators. Central pattern generators are collections of neurons that generate the basic pattern for rhythmic movements. They exhibit common behavioral features such as the generation of rhythmic motor output in the absence of rhythmic input and entrainment to sensory feedback [23, 24]. These basic features are also demonstrated by artificial neural oscillators, systems of nonlinear equations that exhibit oscillatory behavior. Researchers have used a plethora of different mathematical equations to model neural oscillator function [25-33]. The behavior produced when coupling these artificial neural oscillators to a mechanical system is similar for many different specific formulations, suggesting that there are general characteristics of rhythmic neuromechanical systems [34-37].

Several key features of artificial neural oscillators make them desirable for bio-inspired control of robots. They provide robust and versatile control over a wide range of conditions. Neural oscillators produce stable limit cycles that are robust to transient perturbations yet flexible in adapting to sensory feedback [38, 39]. As a result, they can generate locomotion that automatically changes gait and adjusts to perturbations to move effectively through a variable environment $[25,26,28$, 40-43]. Importantly, neural oscillators entrain to the dynamics of the mechanical system, driving the system at its resonant frequency [33, 34, 36, 37]. Theoretically, this could minimize the energetic cost of locomotion for legged robots. It also reduces the need for trajectory planning and precise knowledge of mechanical system properties (e.g., mass, stiffness).

Although several studies have reported how artificial neural oscillators entrain to continuous dynamic systems (such as pendulums), their performance in driving discontinuous hybrid dynamic systems (i.e. spring-mass systems with separate stance and aerial phases) is not established. Hybrid dynamic systems do not have a single resonant frequency because their dynamics shift between governing sets of equations. The dynamics of hybrid systems can be quite complex, prohibiting complete analytical solutions in mathematical models unless many simplifications and constraints are applied [44]. Ijspeert and colleagues demonstrated that an artificial neural oscillator (i.e. Hopf oscillator) can drive a simulated spring-mass hopper with hybrid dynamics [45]. They also found that the adaptive artificial neural oscillator adopts a hopping frequency that maximizes a measure of hopper economy [45]. However, they did not perform a thorough mechanical analysis of their simulation so it is unclear how the neural oscillator interacted with the mechanical dynamics.
We built a simple hopping robot with hybrid dynamics so that we could test the ability of an artificial neural oscillator to adaptively entrain to a hybrid system. Our neuromechanical system consisted of a single reciprocal inhibition oscillator (Matsuoka oscillator) coupled to a hopping robot actuated at a single ankle joint. We used two approaches to test the adaptive capabilities of the artificial neural oscillator. First, we examined whether the entrainment of the oscillator-robot system is robust across changes in two control parameters: descending neural drive and muscle gain. Second, we tested the ability of the neuromechanical system to adapt to variation in mechanical properties by altering the resonant frequency of the stance phase via changes in the in-series and in-parallel spring stiffnesses. We hypothesized that the artificial neural oscillator would drive the mechanical system in resonance for the ground contact phase. The aerial phase has no resonance due to the dynamics of projectile motion.

We chose to test the system response to descending neural drive and muscle gain because these are two control parameters with well-understood physiological analogs. In vertebrate animals, locomotion is initiated by descending neural drive from the mesencephalic locomotor region (MLR) of the midbrain [46, 47]. Increased strength of the descending signal from the MLR results in increased frequency and amplitude of rhythmic motor output with abrupt transitions between gaits. In this manner, the brain can govern speed, amplitude and mode of movement. In our simple neuromechanical system, altering the descending signal strength was a way to test if the robot maintained similar hopping dynamics with changes in general excitation. The second control signal, muscle gain, represents the excitation-contraction coupling between the nervous system and muscle. This relationship can vary under physiological conditions such as muscle fatigue [48, 49]. By altering the muscle gain, we examined the ability of the oscillator to adapt to changes in the neuromechanical transfer dynamics. With this parameter, we could test whether the system maintains similar hopping dynamics with changes in the neuromuscular gain.

\section{Methods}

\section{Hopper design}

We designed the robot to represent general aspects of lower limb mechanics during human hopping. It was composed of wooden foot and shank segments joined by a metal hinge joint with one degree of freedom to simulate a simplified ankle. While a hinge joint does not match the true complexity of the ankle joint, it replicates the primary motion of this joint in the sagittal plane during hopping. Neoprene padding on the foot section provided better grip upon landing and light damping to diminish high frequency vibration. This created a more biologically realistic model of the human foot, which employs considerable tissue padding to cushion foot impact [50]. The robot is stabilized in the medio-lateral plane through its attachment to a wooden supporting beam to limit motion to vertical hopping (figure 1). The top of the shank was secured to the beam by a hinge joint, allowing free motion in the sagittal 


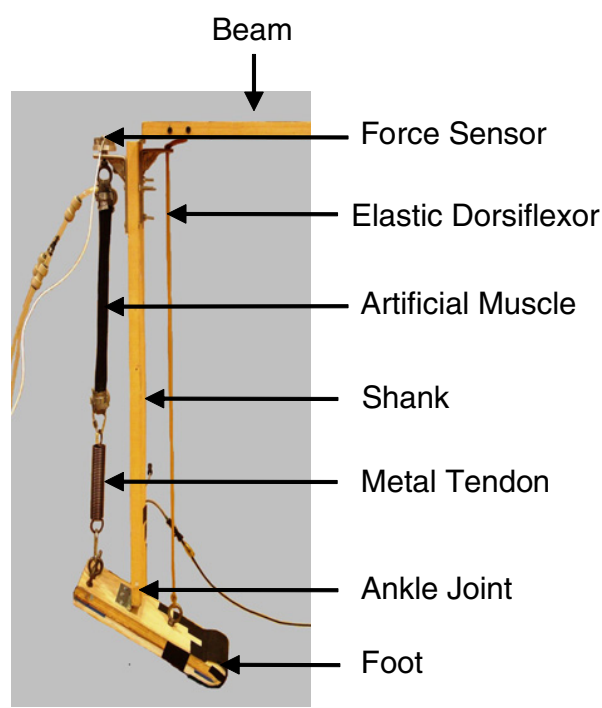

Figure 1. The hopping robot. The artificial muscle was connected to a pneumatic system which regulated air flow into and out of the muscle. The 'tendon' was a metal extension spring. The ankle was a metal hinge joint with a single degree of freedom allowing motion in the sagittal plane.

plane. The beam was attached to a static base through another hinge joint.

The ankle joint was actuated for plantar flexion by an artificial muscle-tendon unit, consisting of a pneumatic muscle and metal extension spring connected in series. The muscle was attached to the proximal shank by an L-bracket and the spring (Century Spring Corp., Los Angeles, CA) inserted via an eyebolt onto the superior, posterior aspect of the foot (figure 1). A single-axis compression load cell (Omegadyne, Sunbury, $\mathrm{OH}$ ), placed between the L-bracket attachment and the superior end of the muscle, allowed measurement of muscle-tendon force over the course of each hop cycle. While the artificial muscle-tendon unit provided ankle plantar flexion, antagonistic dorsiflexion was mediated by a passive spring (elastic band) attached to the anterior side of the shank and foot. We did not include active dorsiflexion for the robot because we wanted the simplest system that could test our hypothesis. Pilot data collections with the robot including an artificial pneumatic muscle controlled by the flexor half-center of the artificial neural oscillator demonstrated qualitatively similar behavior as when an elastic rubber band was used for passive dorsiflexion.

The artificial pneumatic muscle was constructed in the same manner as that used for previous studies on pneumatically powered ankle-foot orthoses [51-53]. The body of the muscle consisted of latex tubing for the inner air bladder and braided polyester sheathing for the outer shell. Four parallel proportional pressure regulators (MAC Valves, Inc., Wixom, MI) supplied compressed air (0-6.2 bar) to each artificial muscle via nylon tubing. An analog-controlled solenoid valve (MAC Valves, Inc., Wixom, MI) was placed in parallel with the air supply tubing to facilitate exhaust. Air was supplied to the artificial muscle through a regulated air supply through standard pneumatic fittings. We controlled the air pressure to

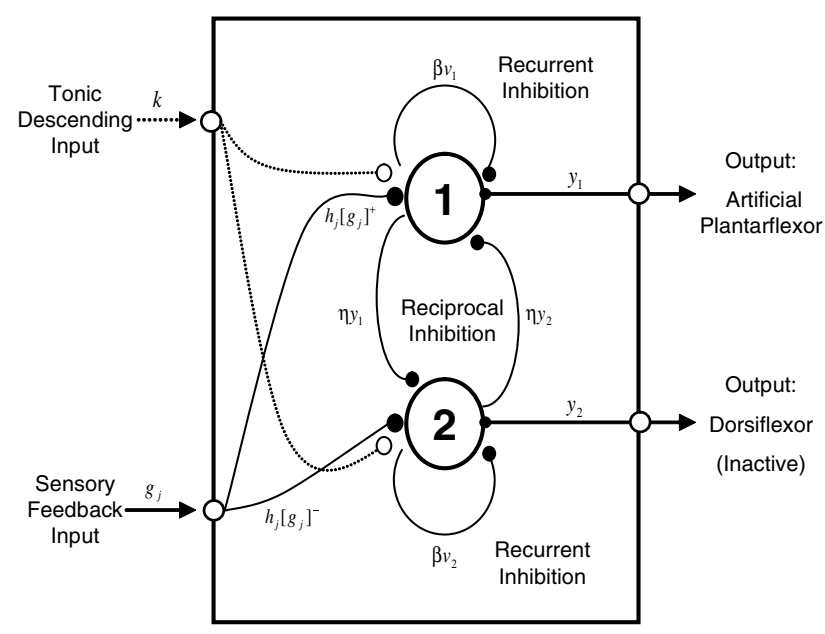

Figure 2. Artificial neural oscillator schematic. Ankle angle served as the sensory feedback signal. Oscillator output included both flexor and extensor muscle activation signals. For our testing, only the plantar flexor oscillator half-center was used. Dorsiflexion on the robot was achieved by a passive rubber spring.

the muscle through a real-time computer interface (dSPACE Inc., Northville, MI; $1000 \mathrm{~Hz}$ ) that received its control signal from the neural oscillator output. The artificial muscle had a similar force bandwidth $(2.4 \mathrm{~Hz})$ as human skeletal muscle $(2.2 \mathrm{~Hz})[52,54]$. The effective mass of the robot was approximately $3.6 \mathrm{~kg}$.

\section{Artificial neural oscillator}

The neural circuitry of a central pattern generator (CPG) was modeled using the dynamic equations for a simplified reciprocal inhibition oscillator developed by Matsuoka (figure 2) [31, 55]. The neural oscillator model was governed by the following set of differential equations:

$$
\begin{aligned}
& \tau_{1} \dot{x}_{1}=-x_{1}-\beta v_{1}-\eta\left[x_{2}\right]^{+}+k-h_{j}\left[g_{j}\right]^{+} \\
& \tau_{2} \dot{v}_{1}=-v_{1}+\left[x_{1}\right]^{+} \\
& \tau_{1} \dot{x}_{2}=-x_{2}-\beta v_{2}-\eta\left[x_{1}\right]^{+}+k-h_{j}\left[g_{j}\right]^{-} \\
& \tau_{2} \dot{v}_{2}=-v_{2}+\left[x_{2}\right]^{+} \\
& y_{i}=\left[x_{i}\right]^{+} \\
& T_{i}=G_{i} y_{i},
\end{aligned}
$$

where $\tau_{1}$ and $\tau_{2}$ are time constants of the inner state and adaptation effect, respectively, which adjust the shape and intrinsic frequency of the oscillator, $\eta$ is a constant that affects the reciprocal inhibition between the two neurons, $\beta$ is a constant involved in the recurrent inhibition of each neuron, $k$ is the tonic descending signal strength and $g$ is the feedback input (i.e. joint angle) with a corresponding gain $h$. The value $x_{i}$ is a discontinuous term, where $\left[x_{i}\right]^{+}$is the positive part of $x_{i}$ and used as the output signal from the oscillator, $y_{i} . \quad T$ is the final control signal (i.e. voltage) sent to the pressure regulator and is the product of $y_{i}$ and muscle gain, $G$. This control signal can be thought of as analogous to an electromyography linear envelope [56]. The base value for each of the model parameters was chosen using guidelines developed by Williamson through a rigorous analysis of the 
Table 1. Base parameter values used for the neural oscillator (unitless).

\begin{tabular}{lc}
\hline Parameter & Value \\
\hline$\tau_{1}$ & 0.02 \\
$\tau_{2}$ & 0.04 \\
$\beta$ & 2.5 \\
$k$ & 3.0 \\
$h$ & 5.0 \\
$G$ & 10.0 \\
\hline
\end{tabular}

controller properties [57, 58] (table 1). The values of $\tau_{1}$ and $\tau_{2}$ were set to provide an intrinsic oscillator frequency approximately one-half the resonant mechanical frequency of the hopper in the standard spring configuration. The precise intrinsic frequency of the oscillator is unlikely to influence the results, however, because this oscillator has been found to robustly entrain to the mechanical system over a wide frequency range [58]. The tonic descending signal represents descending neural drive from the midbrain [46, 47]. Based on the assumption that $k$ would influence system dynamics in a manner consistent with biological data, we expected increased descending drive to produce increased frequency and/or amplitude of motor output. Muscle gain $(G)$ represents the neuromechanical transformation between neural signal and contraction dynamics.

We used the Matsuoka artificial neural oscillator because of its simplicity, demonstrated robustness and adaptability, and wide use in neuromechanical modeling and adaptive robotics [25, 31, 37-43, 55, 57, 59]. However, as discussed in the introduction, previous experiments have found similar system behavior using a number of different oscillators.

\section{Experimental procedure}

Two separate experimental manipulations were performed to evaluate the ability of the neural oscillator to provide robust and adaptive control of the hopping robot. First, we manipulated oscillator control parameters to examine the sensitivity of the neuromechancial system. Second, we manipulated the properties of the mechanical system to examine the ability of the neural oscillator to drive the system over a range of stancephase resonant frequencies. We varied the stiffness of the muscle-tendon unit by altering the arrangement of in-series and in-parallel springs, described in detail below.

In the first experimental manipulation, we varied the tonic descending signal strength $(k)$ and muscle gain $(G)$ parameters and analyzed the resulting system performance. The standard spring setup was used as depicted in figure 1 (tendon spring of $2.7 \mathrm{kN} \mathrm{m}^{-1}$ ). The $k$ and $G$ values were individually varied over a range of 0.1 to 100 . The value of each parameter was increased exponentially, resulting in at least ten trials for each varied parameter. For each trial, all model parameters were fixed except the current variable under investigation (either $k$ or $G$ ) (table 1). With increasing $G$, the hopping dynamics rapidly reached a consistent pattern that was robust to further changes in $G$. Because tonic drive $(k)$ exhibited a less sharp saturation, more values of $k$ were tested to resolve the point at which the hopping dynamics reached a consistent pattern. Kinetic and kinematic data were collected for five continuous seconds after allowing the robot to first reach steady state hopping.

In the second experiment we examined system performance under conditions of varying muscle-tendon stiffness, manipulated by changing the arrangement of springs in parallel and in series. First, we tested four different springs $\left(1.7,2.7,4.1,6.7 \mathrm{kN} \mathrm{m}^{-1}\right)$ in the standard arrangement, changing the in-series stiffness (figure 1 ). The springs were of equal length and differed minimally in mass $(0.147,0.16$, $0.19,0.19 \mathrm{~kg}$ ). In a second spring arrangement, we used the $2.7 \mathrm{kN} \mathrm{m}^{-1}$ spring in series with the artificial muscle and added one of the four springs of differing stiffness in parallel. The second spring was attached (via rigid cables) in parallel to the muscle-tendon unit, so that the two were positioned bisymmetrically about the center point on the posterior side of the shank section. This arrangement of the parallel spring was intended to mimic previous experiments of humans hopping in place with an elastic ankle-foot orthosis [17].

\section{Data collection}

An eight-camera motion analysis system (Motion Analysis, Santa Rosa, CA) was used to record segment and joint kinematics at $120 \mathrm{~Hz}$. We placed reflective markers on the toe and heel of the foot, at the proximal and distal ends of the artificial muscle, at the center of rotation of the ankle joint and in a triad of markers on the anterior face of the shank. We measured the ankle angle in the sagittal plane using an electrogoniometer (Biometric Ltd, Ladysmith, VA). This angle served as the neural oscillator feedback signal, which was the sole input to the computer controller. We positioned the hopper device over a force plate and collected ground reaction force (GRF) data at $1200 \mathrm{~Hz}$ (Advanced Mechanical Technology, Watertown, MA).

\section{Data analysis}

The data were filtered and processed using commercial software programs (Visual3D, C-Motion, Rockville, MD; MATLAB, Mathworks). Data were filtered using a fourthorder zero-lag Butterworth low-pass filter with a $15 \mathrm{~Hz}$ cutoff frequency. Individual hop cycles were defined as the time from ground contact to subsequent ground contact, determined from the GRF data. The data for all complete hop cycles in each $5 \mathrm{~s}$ trial were aligned and averaged at each time point to generate a single average hop cycle. Incomplete hop cycles were excluded from analysis. The number of complete hop cycles in the $5 \mathrm{~s}$ recording period varied between 9 and 14 depending on hop frequency.

\section{Calculations}

For each hopping cycle, we measured and calculated a number of traditional biomechanical variables. We determined hopping frequency, stance duration and aerial phase duration from the ground reaction force data. We calculated the displacement of the tendon spring by dividing the muscletendon force (measured with the load cell) by the spring stiffness. We used kinematic markers on the proximal and 

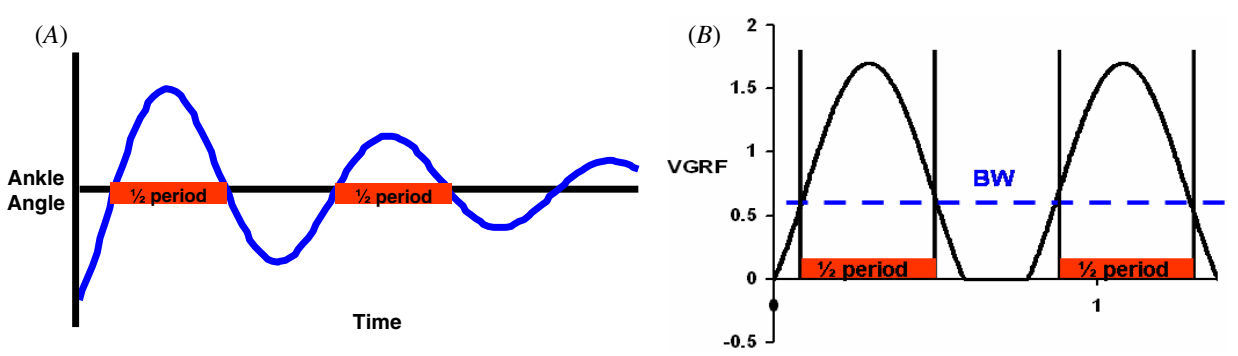

Figure 3. Measurements of the half-period of vibration for passive free vibration without an aerial phase and for active hopping. (A) Example of amplitude of ankle angle versus time in free vibration testing. The time duration of a given cycle half-period is the value of interest. $(B)$ Example of vertical ground reaction force (VGRF) versus time during hopping. Points are identified on each force curve where the data equal bodyweight of the robot. The half-period of vibration during the stance phase is the period of time that the ground reaction force is above the bodyweight threshold.

distal ends of the muscle to record muscle displacement. We calculated the mechanical power of the muscle and tendon by multiplying their respective velocities by the muscle-tendon force. By integrating the power curve with respect to time, we calculated mechanical work. To evaluate the efficiency of the system, we calculated the fraction of total mechanical work done passively through energy cycling in the spring and actively by the artificial muscle.

To test whether the artificial neural oscillator drove the hopper at the stance phase resonant frequency, we compared the half-period of the stance phase (time duration for bodyweight to bodyweight in the ground reaction force) with the half-period measured during free vibration (figure 3). The mathematics for determining the half-period of the stance phase is explained more extensively by Cavagna et al [60]. The mathematics for determining the halfperiod of free vibration is explained more extensively by Tongue [61].

\section{Results}

Changes in the tonic descending drive $(k)$ and muscle gain $(G)$ had similar qualitative effects on hopping mechanics. At values lower than 0.1 for either parameter, the robot did not hop with an aerial phase. Between values of 0.1 and 1 , hopping mechanics were very sensitive to both parameters. The control signal generated by the artificial neural oscillator at these parameter values was of low amplitude and peaked later in the hop cycle (figures 4 and 5). At slightly higher levels of tonic descending drive and muscle gain, the control signal occurred earlier in the hop cycle and saturated in amplitude (figures 4 and 5). As a result of the earlier control signal in the hop cycle, the peak muscle-tendon force, peak ground reaction force and duration of the aerial phase were greater at higher values of tonic descending drive and muscle gain (figures 6 and 7). A longer aerial phase indicates that the robot jumped higher, because the robot undergoes simple ballistic motion in this phase.

Hopping mechanics showed little variation with changes in descending drive or muscle gain over the parameter range of 1-100. For this 100-fold change in parameter values, the artificial muscle developed force while shortening to produce positive power and the tendon underwent stretch and recoil (figures 4 and 5). Stance duration, hopping frequency, peak force and displacement of the ankle, muscle and tendon maintained relatively constant values (figures 6 and 7). Hopping mechanics remain especially constant with altered muscle gain $(G)$. Hopping frequency changed by $5 \%(1.88-$ $1.98 \mathrm{~Hz})$ and ground contact time changed by $4 \%(0.258$ 0.269 s) over this 100 -fold range in $G$ (figure 7 ). Increments in tonic drive $(k)$ over this range leads to small increments in peak muscle-tendon force, and corresponding small increments in aerial time and decrements in hop frequency (figure 6). However, given the 100-fold range in $k$, these changes are modest. Hopping frequency changed by less than $10 \%$ (1.85$2.01 \mathrm{~Hz})$ and ground contact time changed by less than $13 \%$ $(0.234-0.269 \mathrm{~s})$. Over the 100-fold range in both parameters, elastic energy storage in the tendon spring accounted for 70 $79 \%$ of the total mechanical work done by the muscle-tendon system (muscle contributed 21-30\%) (figures 6 and 7).

The neural oscillator consistently drove the robot at the resonant half-period of the stance phase. We manipulated the resonant half-period by changing the stiffness of the inseries spring (figure $8(A)$ ) and by adding a parallel spring of varying stiffnesses (figure $8(B)$ ). This resulted in resonant half-periods ranging between 0.13 and $0.19 \mathrm{~s}$ for the in-series arrangement and 0.12 and $0.16 \mathrm{~s}$ for parallel arrangement. The experimentally measured half-period based on ground force data (figure $3(B)$ ) consistently matched the resonant halfperiod of the robot. The slope of the least squares linear regression fit between these variables was $1.05\left(R^{2}=0.93\right)$ for the single-spring setup and $1.02\left(R^{2}=0.95\right)$ for the parallelspring setup. This indicates that the robot was consistently driven at stance phase resonance.

\section{Discussion}

Hopping dynamics were primarily dependent on the intrinsic mechanical properties of the robot. Muscle gain and supraspinal gain were varied over 100-fold, but the hopping frequency and ground contact time varied by less than 10 and $13 \%$, respectively. The artificial neural oscillator entrained to the natural frequency of the mechanics to achieve stance phase resonance. When the intrinsic mechanical properties of the robot were altered by changing the spring arrangement, the 


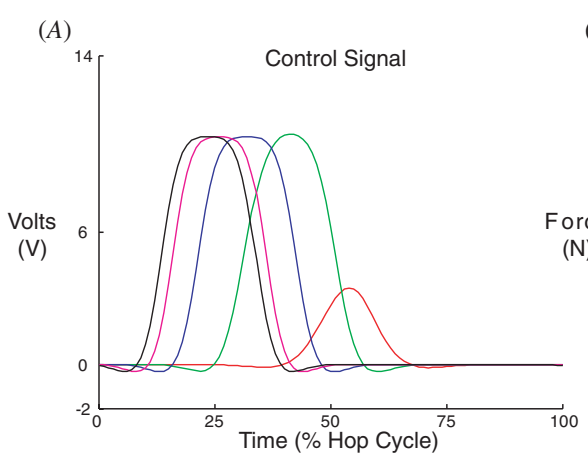

(B)
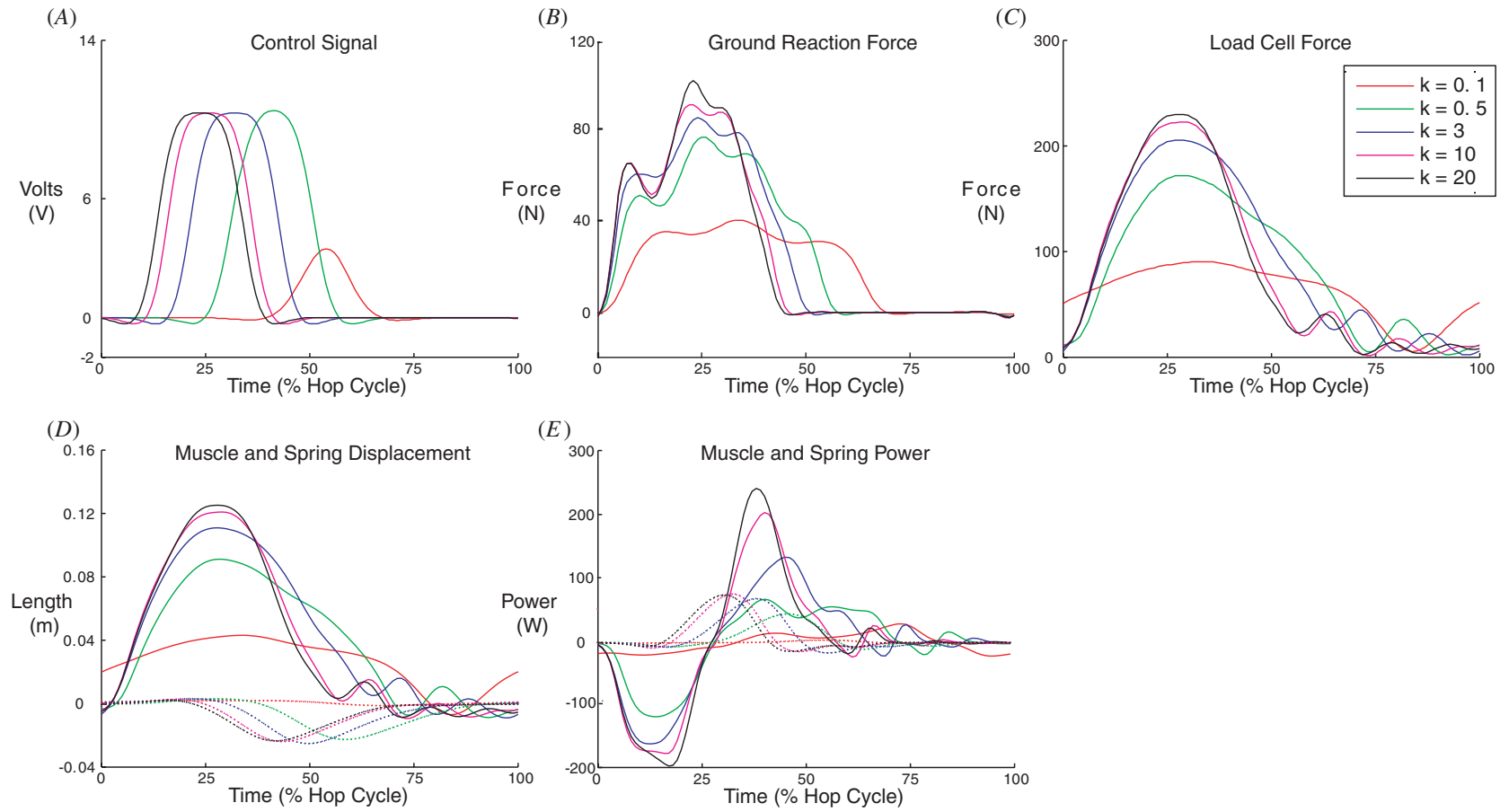

Figure 4. Effect of descending tonic drive $(k)$ on hop cycle dynamics. $(A)$ Muscle control signal, $(B)$ ground reaction force, $(C)$ load cell force, $(D)$ muscle and spring displacement and $(E)$ muscle and spring power. In $(D)$ and $(E)$, solid lines are for the spring and dashed lines are for the muscle. As tonic drive increased, the robot progressed toward consistent hop dynamics that were insensitive to further increases in $k$. Waveforms are the mean of hops in a $5 \mathrm{~s}$ trial. Initial ground contact is at $0 \%$ of the hop cycle.
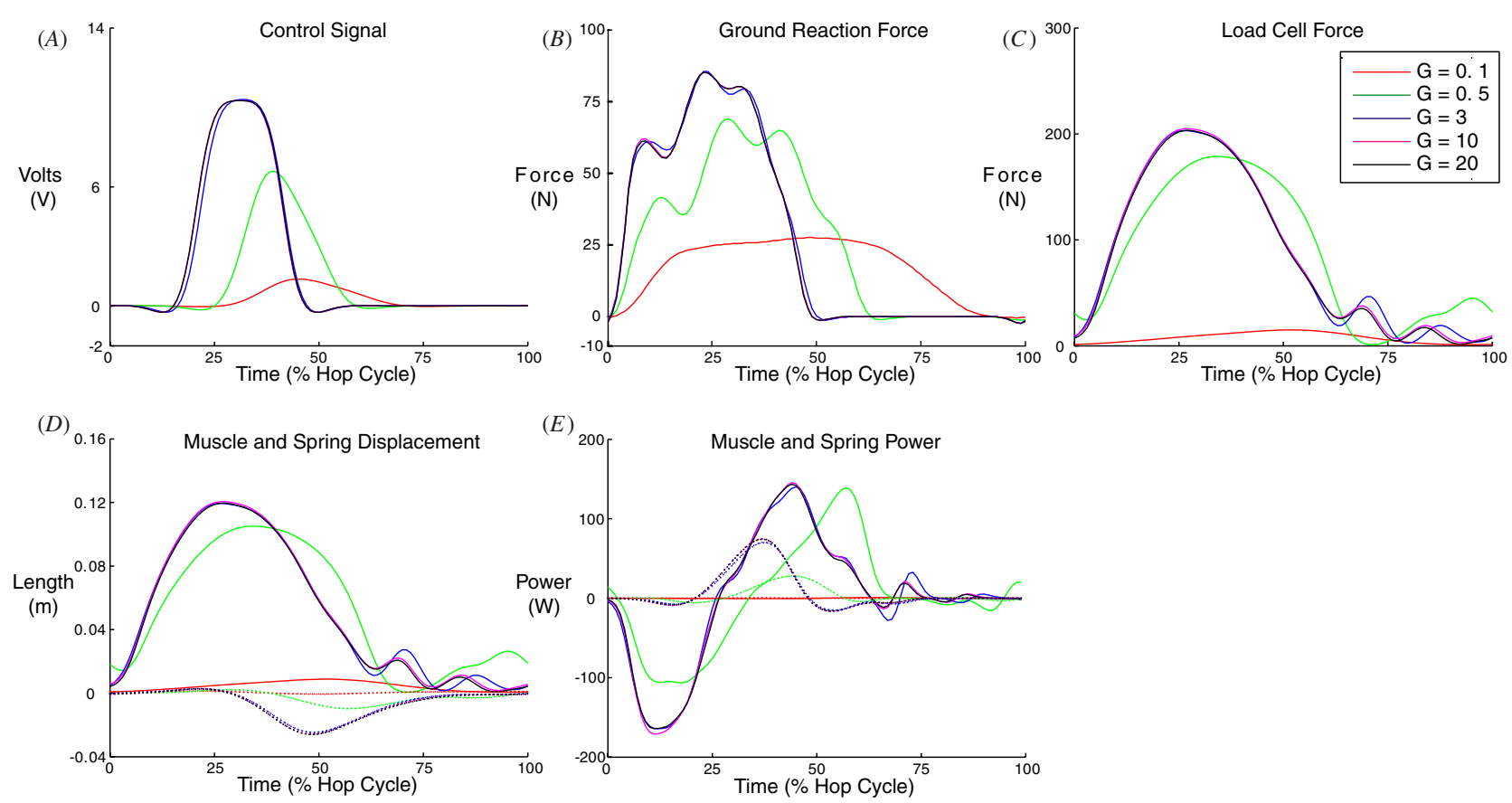

Figure 5. Influence of muscle gain $(G)$ on hop cycle dynamics. $(A)$ Control signal, $(B)$ ground reaction force, $(C)$ load cell force, $(D)$ muscle and spring displacement and $(E)$ muscle and spring power. In $(D)$ and $(E)$, solid lines are for the spring and dashed lines are for the muscle. The robot progressed toward consistent hop dynamics that were insensitive to further increases in $G$. Waveforms are the mean of hops in a $5 \mathrm{~s}$ trial. Initial ground contact is at $0 \%$ of the hop cycle.

neural oscillator consistently drove the system at the resonant half-period of the stance phase (figure 8).
In contrast to our expectations, changes in descending neural drive had little effect on robot hopping dynamics. 
(A)

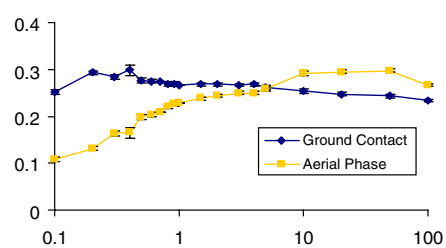

(B)

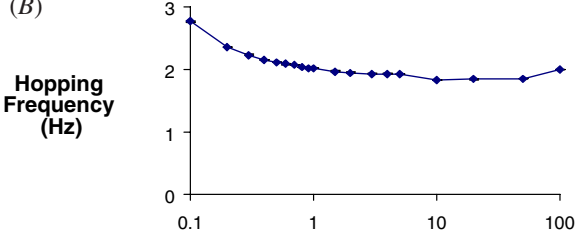

(C)
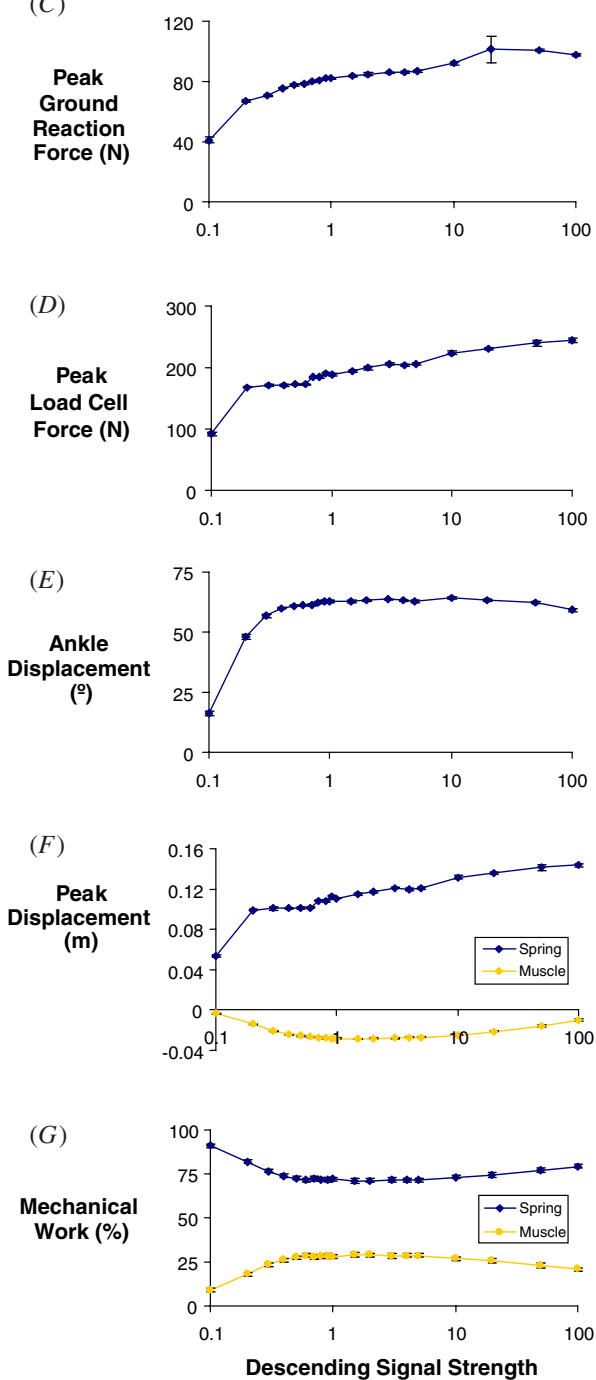

Figure 6. The effect of descending signal strength on hopping mechanics. $(A)$ Ground contact and aerial time, $(B)$ hopping frequency, $(C)$ ground reaction force, $(D)$ load cell force, $(E)$ ankle angular displacement, $(F)$ spring and muscle displacement and $(G)$ spring and muscle mechanical work. Values for descending signal strength are displayed on a $\log _{10}$ axis. Values are the means \pm s.e.m.
(A)
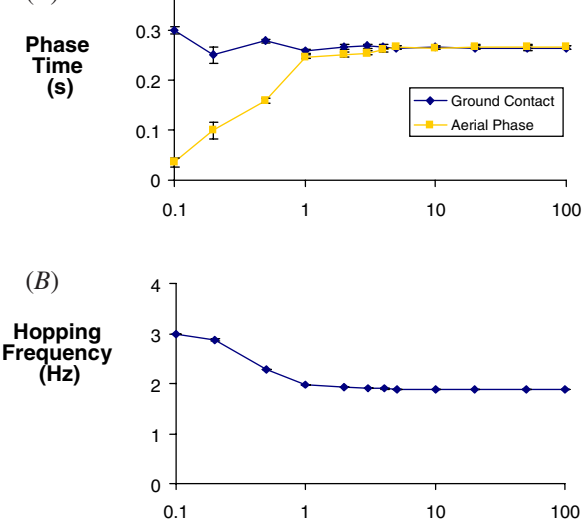

(C)
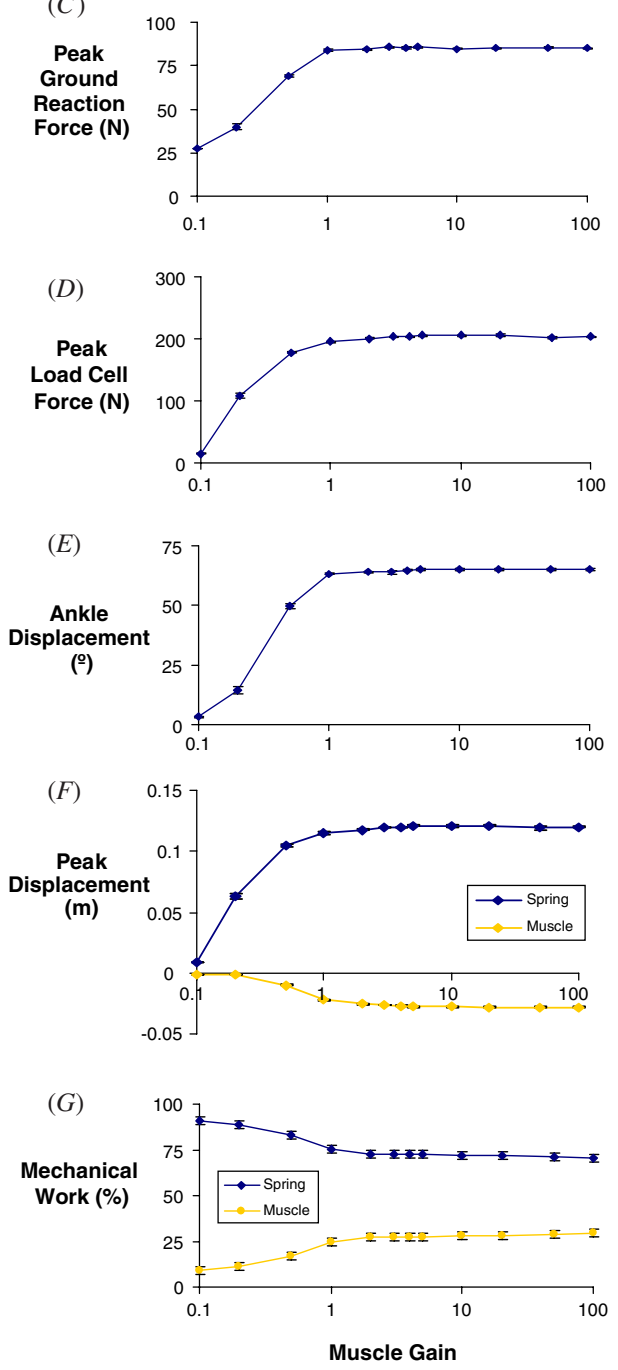

Figure 7. The effect of muscle gain on hopping mechanics. $(A)$ Ground contact and aerial time, $(B)$ hopping frequency, $(C)$ ground reaction force, $(D)$ load cell force, $(E)$ ankle angular displacement, $(F)$ spring and muscle displacement and $(G)$ spring and muscle mechanical work. Values for muscle gain are displayed on a $\log _{10}$ axis. Values are the means \pm s.e.m. 


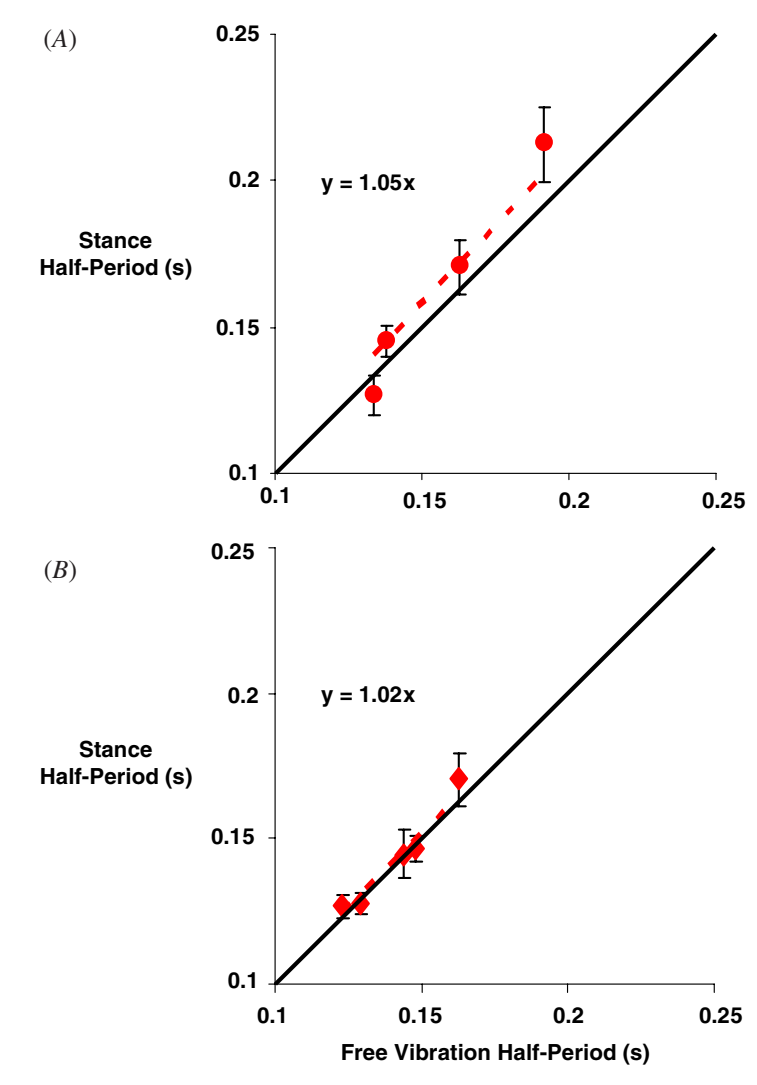

Figure 8. Comparison of half-period of vibration during stance phase with the resonant half-period of free vibration. A line with slope of 1 (solid thick line) is the expected relationship if the robot was driven at stance resonance. The dashed line indicates the least squares linear regression fit of the experimental data. (A) Data acquired using the standard spring setup as shown in figure 1 and changing the stiffness of the spring tendon. $(B)$ Data acquired using a parallel spring in addition to the standard setup of the robot, changing the stiffness of the parallel spring. Values are the means \pm s.e.m.

Studies on vertebrate locomotion have revealed that increases in descending neural drive produce stronger muscle contractions during stance, higher frequency movements and can lead to abrupt transitions in gait $[46,47,80]$. This hybrid dynamic robot does not have a single resonant frequency because the dynamics of the stance and aerial phases are discontinuous. Aerial phase duration depends only on the vertical take-off velocity at the end of stance. We expected that increases in neural drive would increase take-off velocity, resulting in a longer aerial phase and lower hopping frequency. Hopping mechanics were sensitive over a small range of parameter values, but a 100-fold change in the parameter had little effect above the value of 1 . The relative consistency of movement dynamics likely resulted from the constrained mechanical structure. Our robot represented a simple hopping system consisting of one single degree of freedom joint and one actuator. Although gait changes were not possible, it was possible to maintain stance phase resonance while altering aerial phase duration and hopping frequency. It is not clear why the system maintained such consistent dynamics across changes in descending neural drive.
The robot greatly relied on elastic energy storage and return during hopping. At muscle gain and supraspinal gain parameters greater than 1, the ankle extensor muscle did not change length for the first half of stance and then contracted to produce positive work during the second half of stance (figures 4 and 5). In contrast, the tendon lengthened during the first half of stance and shortened during the second half of stance (figures 4 and 5). These behaviors are similar to those of the human triceps surae and Achilles' tendon during hopping and running [62-64].

The robot demonstrated an important difference between its behavior and that of humans. When humans hop with added parallel spring stiffness using an ankle-foot orthosis, they maintain the same ground contact time as they used without the device [17]. In contrast, our robot exhibited variable ground contact times with a similar manipulation. This adjustment allowed the robot to maintain stance phase resonance. Several factors could explain this difference between human and robot, all relating to the greater complexity of the human neuromechanical system.

Humans might maintain stance phase resonance with added parallel elasticity by adjusting their movement patterns subtly rather than altering their ground contact time. The more complex, nonlinear musculoskeletal dynamics of humans and other animals can be altered by limb posture, muscle coactivation and choices in synergist muscle activation [65-70]. The choice of a constant ground contact time both with and without an elastic orthosis may have benefits related to muscle activation dynamics, muscle efficiency or metabolic cost $[5,71-73]$.

Another potential explanation for the difference between human and robot is the simplistic model of the robot nervous system. Humans, like all vertebrates, have a hierarchical neural control architecture that integrates brain function, spinal networks and reflex feedback [74, 75]. In particular, reflexive excitation from stretch and load-dependent proprioceptors contribute substantially to extensor muscle activation during stance [18, 19, 77, 78]. There may be an optimal ground contact time based on the timing of reflex pathways and the inherent system mechanics (e.g. gravity, body mass) for stability [79]. Our results do not provide a definitive explanation for the differences between robot and human, but they do present possibilities for designing future experiments.

An alternative modeling approach for the analysis of bouncing gait dynamics would be to rely entirely on computer simulations. A key advantage to building an actual physical device is that it benefits from accurate 'physics for free'. All computer models and simulations rely on simplifying assumptions about mechanical properties and dynamics. Physical models allow testing and validation of these mathematical models, and often reveal aspects of the system that otherwise would have been missed [81]. For this reason, numerous groups have used robots as research tools for understanding the basic principles of human and animal locomotor mechanics [82-86]. In addition, the ability to both see a system in action and quantify its mechanical performance often provides more convincing evidence for the model than simulation alone. Robotic models serve as an important tool 
for engaging students in research and increasing the impact of biomechanical studies, because robots have widespread appeal and strong presence in popular culture.

Our biologically inspired robot also has important implications for the design and control of robots. Previous work has demonstrated the robust and adaptive performance of neural oscillators for controlling simple resonant systems [33, 36-38]. However, few studies have investigated the dynamics of neural oscillators coupled to hybrid dynamics systems, which do not have a single resonant frequency. A recent study has demonstrated that a simple adaptive neural oscillator model can drive a hopping quadrupedal robot at its resonant frequency [45]. However, no previous study has included a detailed analysis of locomotor mechanics while driven by a neural oscillator. Our results demonstrate that the Matsuoka oscillator drives the mechanical system at the ground contact resonance during hopping (figure 8). Analysis of locomotor mechanics reveals that this allows approximately $75 \%$ of the mechanical work to be done by passive energy cycling in the tendon spring (figures 6 and 7). This result suggests that neural oscillator control, when appropriately coupled to the mechanical system, could improve efficiency in a wide array of robotic applications. Furthermore, the neural oscillator was able to adapt to the change in spring stiffness without the need for hand tuning of control parameters (figure 8). This is consistent with previous studies that suggest that neural oscillators produce stabile motor output that eliminates the need for trajectory planning and precise knowledge of mechanical system properties [25, 33, 38, 39, $41,45]$. Thus, neural oscillators can provide robust, adaptable and economic control of hybrid dynamic systems.

One potential limitation to artificial neural oscillator control is that some behaviors require motion at non-resonant frequencies. However, networks of coupled neural oscillators can allow a wider range of behaviors and locomotor modes, with gait transitions automatically controlled by system dynamics [26, 28]. Additionally, a hierarchical control structure could allow the neural oscillator rhythm to be adjusted by higher level control, as observed in animals [74, 75]. Such control architectures, although more complex, would still reap the benefits of stable oscillator dynamics: automatic entrainment and integration of sensory feedback, and economic locomotion without trajectory planning.

\section{Conclusion}

Biomimetic robots help reveal basic principles of neuromechanical integration and provide insight into human and animal movement. The very simple neuromechanical hopping system studied here demonstrates the ability of artificial neural oscillators to provide robust, adaptive and economic control of hybrid dynamic systems. More anthropomorphic robot designs with similar control architecture could be important tools to investigate the neuromechanical integration for dynamically stable running. Yet, addition of multiple joints, actuators and neural oscillators will immensely increase the system complexity. The greatest insight is gained from neuromechanical models that incorporate the minimal complexity necessary for the desired system behavior. Consequently, an important goal of future work will be to identify the minimal neuromechanical system necessary to obtain stable forward bouncing locomotion.

\section{Acknowledgments}

The authors would like to thank Catherine Kinnaird and Tom Serbowicz for their assistance with the project. This work was supported by an NSF CAREER award to DPF (BES-0347479), an NIH grant to DPF (R01 NS45486), an NSF Bioinformatics Postdoctoral Fellowship to MAD (DBI-0630664) and the University of Michigan UROP.

\section{References}

[1] Cavagna G A, Heglund N C and Taylor C R 1977 Mechanical work in terrestrial locomotion: two basic mechanisms for minimizing energy expenditure Am. J. Physiol. 233 R243-61

[2] Full R J and Farley C T 2000 Musculoskeletal dynamics in rhythmic systems: a comparative approach to legged locomotion Biomechanics and Neural Control of Posture and Movement ed J M Winters and P E Crago (New York: Springer-Verlag)

[3] Biewener A A 2006 Patterns of mechanical energy change in tetrapod gait: pendula, springs and work J. Exp. Zool. 305A 899-911

[4] McMahon T A and Cheng G C 1990 The mechanics of running: how does stiffness couple with speed? J. Biomech. 23 65-78

[5] Kram R and Taylor C R 1990 Energetics of running: a new perspective Nature 346 265-7

[6] Kram R 2000 Muscular force or work: what determines the metabolic energy cost of running? Exerc. Sport Sci. Rev. 28 $138-43$

[7] Taylor C R, Heglund N C, McMahon T A and Looney T R 1980 Energetic cost of generating muscular force during running: a comparison of small and large animals J. Exp. Biol. 86 9-18

[8] Farley C T and McMahon T A 1992 Energetics of walking and running - insights from simulated reduced-gravity experiments J. Appl. Physiol. 73 2709-12

[9] Alexander R M and Bennet-Clark H C 1977 Storage of elastic strain-energy in muscle and other tissues Nature 265 114-7

[10] Alexander R M 1988 Elastic Mechanisms in Animal Movement (Cambridge: Cambridge University Press)

[11] Farley C T, Blickhan R, Saito J and Taylor R C 1991 Hopping frequency in humans: a test of how springs set stride frequency in bouncing gaits J. Appl. Physiol. 71 2127-32

[12] Farley C T and Gonzalez O 1996 Leg stiffness and stride frequency in human running J. Biomech. 29 181-6

[13] He J P, Kram R and McMahon T A 1991 Mechanics of running under simulated low gravity J. Appl. Physiol. 71 863-70

[14] Ferris D P and Farley C T 1997 Interaction of leg stiffness and surface stiffness during human hopping J. Appl. Physiol. 82 15-22

[15] Ferris D P, Louie M and Farley C T 1998 Running in the real world: adjusting leg stiffness for different surfaces Proc. $R$. Soc. Lond. 265 989-94

[16] Ferris D P, Liang K and Farley C T 1999 Runners adjust leg stiffness for their first step on a new running surface J. Biomech. 32 787-94

[17] Ferris D P, Bohra Z A, Lukos J R and Kinnaird C R 2006 Neuromechanical adaptation to hopping with an elastic ankle-foot orthosis J. Appl. Physiol. 100 163-70 
[18] Melville-Jones G and Watt D G 1971 Observations on the control of stepping and hopping movements in man J. Physiol. 219 709-27

[19] Dietz V, Schmidtbleicher D and Noth J 1979 Neuronal mechanisms of human locomotion J. Neurophysiol. 42 1212-22

[20] Cavagna G A 1970 Elastic bounce of the body J. Appl. Physiol. 29 279-82

[21] Takeshita D, Shibayama A, Muraoka T, Muramatsu T, Nagano A, Fukunaga T and Fukashiro S 2006 Resonance in the human medial gastrocnemius muscle during cyclic ankle bending exercise J. Appl. Physiol. 101 111-8

[22] Bach T M, Chapman A E and Calvert T W 1983 Mechanical resonance of the human body during voluntary oscillations about the ankle joint J. Biomech. 16 85-90

[23] Pearson K G 1993 Common principles of motor control in vertebrates and invertebrates Annu. Rev. Neurosci. 16 265-97

[24] Grillner S and Wallen P 1985 Central pattern generators for locomotion, with special reference to vertebrates Annu. Rev. Neurosci. 8 233-61

[25] Taga G, Yamaguchi Y and Shimizu H 1991 Self-organized control of bipedal locomotion by neural oscillators in unpredictable environment Biol. Cybern. 65 147-59

[26] Collins J J and Richmond S A 1994 Hard-wired central pattern generators for quadrupedal locomotion Biol. Cybern. $71375-85$

[27] Buchli J, Righetti L and Ijspeert A J 2006 Engineering entrainment and adaptation in limit cycle systems-from biological inspiration to applications in robotics Biol. Cybern. 95 645-64

[28] Ijspeert A J, Crespi A, Ryczko D and Cabelguen J M 2007 From swimming to walking with a salamander robot driven by a spinal cord model Science 315 1416-20

[29] Kopell N and Ermentrout G B 1988 Coupled oscillators and the design of central pattern generators Math. Biosci. 90 87-109

[30] Ghigliazza R M and Holmes P 2004 A minimal model of a central pattern generator and motoneurons for insect locomotion Siam J. Appl. Dyn. Syst. 3 671-700

[31] Matsuoka K 1985 Sustained oscillations generated by mutually inhibiting neurons with adaptation Biol. Cybern. $52367-76$

[32] Skinner F K, Kopell N and Marder E 1994 Mechanisms for oscillation and frequency control in reciprocally inhibitory model neural networks J. Comput. Neurosci. $169-87$

[33] Hatsopoulos N G 1996 Coupling the neural and physical dynamics in rhythmic movements Neural Comput. 8 $567-81$

[34] Iwasaki T and Zheng M 2006 Sensory feedback mechanism underlying entrainment of central pattern generator to mechanical resonance Biol. Cybern. 94 245-61

[35] Sekerli M and Butera R J 2005 Oscillations in a simple neuromechanical system: underlying mechanisms J. Comput. Neurosci. 19 181-97

[36] Simoni M F and DeWeerth S P 2007 Sensory feedback in a half-center oscillator model IEEE Trans. Biomed. Eng. 54 193-204

[37] Verdaasdonk B W, Koopman H and Van Der Helm F C T 2006 Energy efficient and robust rhythmic limb movement by central pattern generators Neural Netw. $19388-400$

[38] Verdaasdonk B W, Koopman H and Van der Helm F C T 2007 Resonance tuning in a neuro-musculo-skeletal model of the forearm Biol. Cybern. 96 165-80

[39] Williamson M M 1998 Neural control of rhythmic arm movements Neural Netw. 11 1379-94

[40] Taga G 1994 Emergence of bipedal locomotion through entrainment among the neuro-musculo-skeletal system and the environment Physica D 75 190-208
[41] Taga G 1995 A model of the neuro-musculo-skeletal system for human locomotion: II. Real-time adaptability under various constraints Biol. Cybern. 73 113-21

[42] Taga G 1995 A model of the neuro-musculo-skeletal system for human locomotion: I. Emergence of basic gait Biol. Cybern. 73 97-111

[43] Taga G 1998 A model of the neuro-musculo-skeletal system for anticipatory adjustment of human locomotion during obstacle avoidance Biol. Cybern. 78 9-17

[44] Holmes P, Full R J, Koditschek D and Guckenheimer J 2006 The dynamics of legged locomotion: models, analyses, and challenges Siam Rev. 48 207-304

[45] Buchli J, Righetti L and Ijspeert A J 2005 A dynamical systems approach to learning: a frequency-adaptive hopper robot Adv. Artif. Life, Proc. 210-20

[46] Steeves J D and Jordan L M 1980 Localization of a descending pathway in the spinal cord which is necessary for controlled treadmill locomotion Neurosci. Lett. 20 283-8

[47] Shik M L, Severin F V and Orlovskii G N 1966 Control of walking and running by means of electric stimulation of the midbrain Biofizika 11 659-66

[48] Westerblad H, Lee J A, Lannergren J and Allen D G 1991 Cellular mechanisms of fatigue in skeletal muscle Am. $J$. Physiol. 261 C195-209

[49] Bigland-Ritchie B $1981 \mathrm{EMG}$ /force relations and fatigue of human voluntary contractions Exerc. Sport Sci. Rev. $975-117$

[50] Aerts P, Ker R F, De Clercq D, Ilsley D W and Alexander R M 1995 The mechanical properties of the human heel pad: a paradox resolved J. Biomech. 28 1299-308

[51] Ferris D P, Czerniecki J M and Hannaford B 2005 An ankle-foot orthosis powered by artificial pneumatic muscles J. Appl. Biomech. 21 189-97

[52] Gordon K E, Sawicki G S and Ferris D P 2006 Mechanical performance of artificial pneumatic2006 muscles to power an ankle-foot orthosis J. Biomech. 39 1832-41

[53] Gordon K E and Ferris D P 2007 Learning to walk with a robotic ankle exoskeleton J. Biomech. 40 2636-44

[54] Aaron S L and Stein R B 1976 Comparison of an EMG-controlled prosthesis and the normal human biceps brachii muscle Am. J. Phys. Med. 55 1-14

[55] Matsuoka K 1987 Mechanisms of frequency and pattern control in the neural rhythm generators Biol. Cybern. $56345-53$

[56] Winter D A 1984 Pathologic gait diagnosis with computer-averaged electromyographic profiles Arch. Phys. Med. Rehabil. 65 393-8

[57] Williamson M M 2003 Oscillators and crank turning: exploiting natural dynamics with a humanoid robot arm Phil. Trans. R. Soc. A 361 2207-23

[58] Williamson M M 1999 Robot arm control exploiting natural dynamics PhD Thesis Massachusetts Institute of Technology, Cambridge

[59] Fukuoka Y, Kimura H and Cohen A H 2003 Adaptive dynamic walking of a quadruped robot on irregular terrain based on biological concepts Int. J. Robot. Res. 22 187-202

[60] Cavagna G A, Franzetti P, Heglund N C and Willems P 1988 The determinants of the step frequency in running, trotting and hopping in man and other vertebrates J. Physiol. 399 81-92

[61] Tongue B H 1996 Principles of Vibration (New York: Oxford University Press)

[62] Lichtwark G A and Wilson A M 2005 In vivo mechanical properties of the human Achilles tendon during one-legged hopping J. Exp. Biol. 208 4715-25

[63] Lichtwark G A and Wilson A M 2006 Interactions between the human gastrocnemius muscle and the Achilles tendon during incline, level and decline locomotion J. Exp. Biol. 209 4379-88 
[64] Lichtwark G A, Bougoulias K and Wilson A M 2007 Muscle fascicle and series elastic element length changes along the length of the human gastrocnemius during walking and running J. Biomech. 40 157-64

[65] Moritz C T and Farley C T 2004 Passive dynamics change leg mechanics for an unexpected surface during human hopping J. Appl. Physiol. 97 1313-22

[66] Carrier D R, Heglund N C and Earls K D 1994 Variable gearing during locomotion in the human musculoskeletal system Science $\mathbf{2 6 5}$ 651-3

[67] Biewener A A 1989 Scaling body support in mammals: limb posture and muscle mechanics Science 245 45-8

[68] Hogan N 1985 The mechanics of multi-joint posture and movement control Biol. Cybernetics 52 315-31

[69] Nichols T R 2002 The contributions of muscles and reflexes to the regulation of joint and limb mechanics Clin. Orthop. Relat. Res. 403 S43-50

[70] Ting L H and Macpherson J M 2005 A limited set of muscle synergies for force control during a postural task J. Neurophysiol. 93 609-13

[71] Lichtwark G A and Wilson A A 2005 Effects of series elasticity and activation conditions on muscle power output and efficiency J. Exp. Biol. 208 2845-53

[72] Lichtwark G A and Wilson A M 2007 Is Achilles tendon compliance optimised for maximum muscle efficiency during locomotion? J. Biomech. 40 1768-75

[73] Hof A L 2003 Muscle mechanics and neuromuscular control J. Biomech. 36 1031-8

[74] Loeb G E, Brown I E and Cheng E J 1999 A hierarchical foundation for models of sensorimotor control Exp. Brain Res. 126 1-18
[75] Nishikawa K et al 2007 Neuromechanics: an integrative approach for understanding motor control Integr. Comp. Biol. 47 16-54

[76] Pearson K G 2004 Generating the walking gait: role of sensory feedback Prog. Brain Res. 143 123-9

[77] Dietz V and Duysens J 2000 Significance of load receptor input during locomotion: a review Gait Posture 11 102-10

[78] Donelan J M and Pearson K G 2004 Contribution of sensory feedback to ongoing ankle extensor activity during the stance phase of walking Can. J. Physiol. Pharmacol. 82 589-98

[79] Geyer H, Seyfarth A and Blickhan R 2003 Positive force feedback in bouncing gaits? Proc. R. Soc. B $2702173-83$

[80] Grillner S, Georgopoulos A P and Jordan L M 1997 Selection and initiation of motor behaviour Neurons, Networks and Motor Behavior ed P S G Stein, S Grillner, A Selverston and D G Stuart (Cambridge: MIT Press) pp 3-19

[81] Alexander R M 2003 Modelling approaches in biomechanics Phil. Trans. R. Soc. 358 1429-35

[82] McGeer T 1990 Passive bipedal running Proc. R. Soc. B240 107-34

[83] McGeer T 1990 Passive dynamic walking Int. J. Robot. Res. $962-82$

[84] Collins S, Ruina A, Tedrake R and Wisse M 2005 Efficient bipedal robots based on passive-dynamic walkers Science 307 1082-5

[85] Raibert M H and Sutherland I E 1983 Machines that walk Sci. Am. 248 44-53

[86] Blickhan R, Seyfarth A, Geyer H, Grimmer S, Wagner H and Gunther M 2007 Intelligence by mechanics Phil. Trans. A 365 199-220 